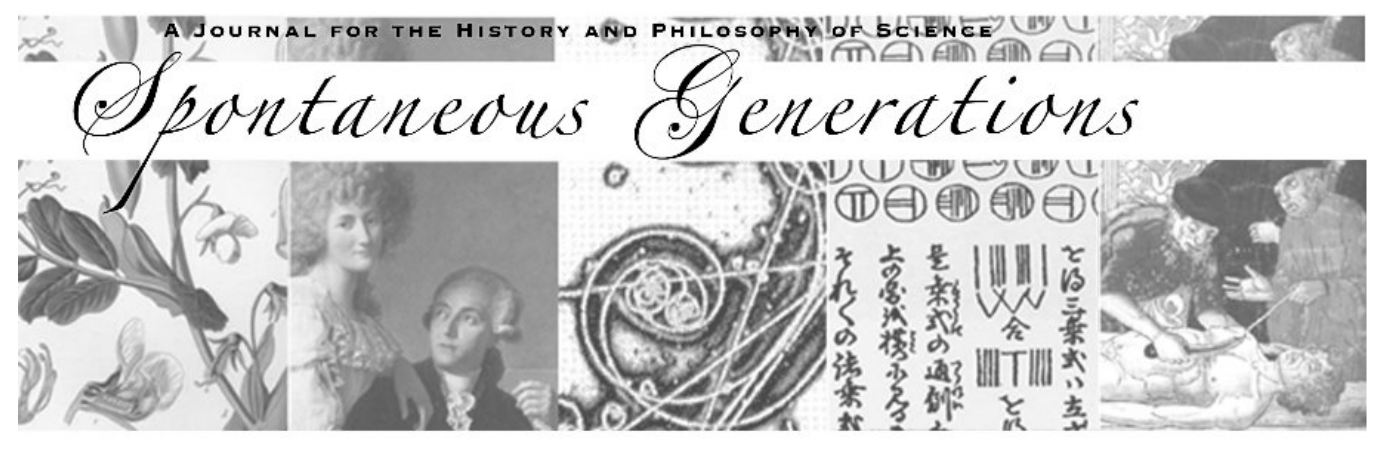

Scientific Instruments: Knowledge, Practice, and Culture

Author(s): Isaac Record

Source: Spontaneous Generations: A Journal for the History and Philosophy of Science, Vol. 4, No. 1 (2010) 1-7.

Published by: The University of Toronto

DOI: $10.4245 /$ sponge.v4i1.14231

EDITORIAL OFFICES

Institute for the History and Philosophy of Science and Technology Room 316 Victoria College, 91 Charles Street West

Toronto, Ontario, Canada M5S 1K7

hapsat.society@utoronto.ca

Published online at jps.library.utoronto.ca/index.php/SpontaneousGenerations ISSN 19130465

Founded in 2006, Spontaneous Generations is an online academic journal published by graduate students at the Institute for the History and Philosophy of Science and Technology, University of Toronto. There is no subscription or membership fee. Spontaneous Generations provides immediate open access to its content on the principle that making research freely available to the public supports a greater global exchange of knowledge. 


\title{
FOCUSED DISCUSSION
}

\author{
Scientific Instruments \\ Knowledge, Practice, and Culture [Editor's Introduction]
}

\author{
Isaac Record*
}

\begin{abstract}
To one side of the wide third-floor hallway of Victoria College, just outside the offices of the Institute for the History and Philosophy of Science and Technology, lies the massive carcass of a 1960s-era electron microscope. Its burnished steel carapace has lost its gleam, but the instrument is still impressive for its bulk and spare design: binocular viewing glasses, beam control panel, specimen tray, and a broad work surface. Edges are worn, desiccated tape still feebly holds instructive reminders near control dials; this was once a workhorse in some lab. But it exists now out of time and place; like many of the scientific instruments we study, it has not been touched by knowing hands in decades.
\end{abstract}

The microscope in the hallway of the IHPST is a metaphor for the place of instruments in science studies. They are of central interest, but they do not really have their own place. Science studies, owing to roots in the history of ideas, conceptual and textual analysis, and ethnography, sometimes struggles to do justice to material things. It is no wonder we so often speak of instruments as theories instantiated, as inscription devices, or as actors in a network-that is, as extensions or modifications of things we already know how to study. But instruments are not those things, and treating them as such could distort our understanding of instruments and their role in science.

\section{WHAT ARE INSTRUMENTS?}

There is no general agreement as to what counts as a "scientific instrument." The term came into wide use only in the nineteenth century and, following Maxwell, indicated "a piece of apparatus constructed specially for the performance of experiments" (quoted in Warner 1990, 88). Seventeenth-century microscopes and air pumps were called "engines," not instruments, but as natural philosophers shifted their methods toward

* Isaac Record is a PhD candidate at the Institute for the History and Philosophy of Science at the University of Toronto. His research is focused on the question of how the adoption and use of scientific instruments-such as the computer-have constrained and shaped scientific practice. Isaac is the current editor of Spontaneous Generations. 
mathematical and geometrical demonstration-and away from ignoble manual labour-makers of such devices began to call them "philosophical." The recent turn toward instruments in HPS relies on an implicit (sometimes explicit) rejection of this distinction. As a result engineers and scientists are now appearing on equal footing in at least some accounts of the history of science (see Galison 1998 for one important example), and Patrick Carroll-Burke advocates a return to explicitly acknowledging the "engine"-aspect of instruments, treating them as "epistemic engines," hard at work variously transducing the world into number, framing phenomena to the senses, generating inscriptions, or "vexing phenomena" within "chambers," spaces "within which phenomena can be materially secured and manipulated" (Carroll-Burke 2001, 607).

In this issue of Spontaneous Generations, several of our contributors wrestle with the notion of instrument, pushing it in sometimes unexpected directions. INGRID HEHMEYER finds that sometimes an instrument isn't an instrument at all-it has been faked. She recounts the tale of one such instrument, an astrolabe, and how it was acquired by Toronto's Royal Ontario Museum and eventually revealed as a forgery. Even when provenance is sure, it is sometimes difficult to determine what should count as an instrument. Maxwell's notion-apparatus for experiment-reflects our intuitions, at least for the physics lab bench. What about the social sciences? Here, in addition to electromechanical devices, human beings themselves become a part of the designed experiment. People playing such roles are called confederates, since (some part of) their behavior is designed by the experimentalist. MAARTEN DERKSEN considers the fundamental tension in using people as instruments, a role that defines them both as free autonomous subjects and as predictable natural objects. When social scientists use people as instruments, "their instrumentality is hidden [from subjects] in order to be effective" (p. 21).

Where social sciences rely on obfuscation, experimentation in the physical sciences can risk equivocating between experimental systems and their target systems outside of the laboratory walls. ROM HARRÉ proposes to distinguish two kinds of equipment for experimentation: "instruments," which are related causally to phenomena of interest, and "apparatus," which are related conceptually to phenomena of interest. Both would have counted as instruments for Maxwell, but Harré's principled distinction implies that each type should be understood differently.

WENDY PARKER asks what one kind of instrument, the digital computer, is for. Many instruments enhance our natural perceptual abilities or help us carry out interventions. Computers seem not, strictly speaking, to do those things, except perhaps in the mundane sense of automating data analysis and fine control of machinery. But we increasingly use computers 
for exploring models, developing explanations, forecasting, designing and interpreting experiments, and by some accounts, even measuring (at any rate, they sometimes "serve the same function as measuring devices, i.e. they can reliably deliver information about real-world target systems that we might otherwise seek using traditional measuring devices" [p. 42]). In order to understand instruments, we must more fully understand each of the roles we ask them to play.

\section{INSTRUMENTS FOR MEASUREMENT AND CONTROL}

Five of our contributors take up the topic of instruments used for measurement and control. The first two deal with the development and maintenance of standards. MICHAEL BARANY recounts an episode from Charles Piazzi Smyth's expedition to measure the pyramids in which Piazzi Smyth abandons a theoretically vetted but practically unusable measurement system in favor of an improvised system resting on the material stability of a hunk of basalt. He concludes that instrumental science is irreducibly local and bottom-up, despite appearances to the contrary; moreover, "the rigors of the basalt Standard's construction undergirded its claims to validity, even as they undermined any possible aspirations to precision or perfection" (p. 57).

JAMES HULL comes to a similar conclusion in his study of the "freeness tester," a control device designed by a Canadian government facility at McGill University. In this case, however, the tester was adopted as an international standard for industrial control by the pulp and paper industry, which "allows a single laboratory in suburban Montreal to measure locally and control globally" (p. 68). Of course, most precision instruments now trace back to national standards laboratories rather than small university facilities, but the point remains: although the construction of standards is a fundamentally local process, it has global implications, including the calibration routines experimentalists perform in order to know their instruments are working properly.

One important question for scientists and philosophers of science is how we can tell when instruments are working. Scientists' claims and philosophers' judgements about the capabilities of instruments are only valid for instruments that are working properly. In this issue, ALLAN FRANKLIN asks the converse question: "How can one determine if an experimental apparatus is giving an incorrect result, if it is speaking falsely?" (p. 71). Elsewhere, Franklin has detailed an epistemology of experiment, a set of strategies used by scientists to ensure apparatuses are working. If an instrument fails to pass one of these tests, he argues, it is unreliable. But what happens when some of these tests are unavailable? In this article, Franklin discusses a case in which certain results remained 
indecisive for fifteen years-until a new apparatus was available to eliminate a plausible alternative explanation.

MARIO BUNGE further complicates any hope of a simplistic account of the operation of instruments. "Most instruments ... do not show directly the value of a property: what they show instead is the value of an indicator, that is an observable counterpart to an unperceivable item" (p. 86). Indicators bridge noumena and phenomena. DAVIS BAIRD, too, notes that indicators are often quite distant from the variables we try to control, and in addition, "the variables one chooses matter" (p. 108). Baird is concerned not only with the security of epistemic claims but also the world we subtly choose to inhabit. We live in a world increasingly manufactured and managed, and this engineered reality goes far beyond simple consumer products. When we set out to measure performance or alter undesired behaviours, we also redefine what it means to be a normal, healthy, intelligent human being. Have we made the right choices?

\section{INSTRUMENTS AS EXTENSIONS}

Much epistemology of science has focused on what we should (or shouldn't) believe about the output of scientific instruments. For example, when, if ever, do instrument outputs give us knowledge about their targets? Van Fraassen has long held that we should accept (but not believe in the existence of) any "unobservable" entities on which we rely to make our theories empirically adequate (1980). On the other hand, Hacking thinks that when experimentalists use "unobservable" entities to investigate further phenomena, the experimentalists are treating those entities as real, and we should follow their lead (1983).

More recently, Humphreys has argued that the traditional distinction between "observable" and "unobservable" is epistemically uninteresting, relying on an arbitrary, anthropocentric distinction. Instruments extend perceptual and cognitive capacities (2004). In this issue, PAUL HUMPHREYS argues that we need to grow our epistemology to match. "There are two options: expanding human frameworks and off-loading some or most of the representational work to artificial cognizers" ( $p$. 113). Two of our contributors make inroads in these directions. MATHIEU CHARBONNEAU integrates the lessons of Clark and Chalmers' extended cognition with Davis Baird's thing knowledge to produce a novel and

attractive account of instruments as materially-extended beliefs existing outside of, but interestingly connected to, the minds of their users. Charbonneau offers the example of an orrery, a device used to calculate and depict the motions of planetary bodies around a central sun. "The orrery [can play] the same functional role for [one] scientist that the astronomical bodies' relative position and trajectory formulas [would] for 
[another]" (p. 125). Indeed, we can consider the orrery to instantiate a set of beliefs about planetary positions, and the proper use of the device is functionally equivalent to remembering those beliefs and putting them to use. PHILIP MCCULLOUGH also takes up the extended mind thesis, arguing that it provides for a new understanding of machine thought, one that survives the test of Searle's Chinese Room thought experiment.

On the other hand, HARRY COLLINS urges caution regarding accounts that treat instruments as having parity with their human users. Implicating both extended mind and actor network theory, Collins argues that such accounts cannot overcome one key distinction between instruments and humans: only humans are embedded in language communities. Instruments have users, and it is these users who determine the meaning of instrument results.

DIFFERENT USERS, DIFFERENT VIEWS

TREVOR LEVERE reviews the ways in which various individuals-collectors and dealers, curators, historians, instrument makers, philosophers, and scientists-come into contact with instruments, and the array of expertise they bring to the conversation about instruments. The nature of history and philosophy of science has been such that we have not always attended to the interests of hobbyists, those instrument users who are not professional scientists. JESSICA ELLEN SEWELL and ANDREW JOHNSTON examine the Dobsonian telescope, an inexpensive but powerful device built from off-the-shelf parts. Sewell and Johnston bring to bear considerations of material culture, highlighting some of the aspects of instrument use often overlooked by science studies, for example, issues of access, the "gesture training" offered by the physical form of the device, and science popularization.

FRANÇOIS THOREAU and MARIA NEICU apply Galison's concept of trading zones to a research group adopting a new shared microscope, and the role this instrument played in the reconfiguring of their research practices and ethics. They identify three repertoires for forming the trading zone: scientific, the microscope was expected to provide better results; economic, researchers felt pressure to use the machine before it outlived the competitive advantage it conferred; and strategic, researchers viewed a properly configured microscope as a "publication machine."

ULJANA FEEST shows that instrument epistemology can be helpful in understanding other aspects of scientific practice. She asks "how do we make sense of concept use in cases where scientists investigate phenomena or objects they don't know much about, or perhaps aren't even sure that they really exist?" (p. 173). Feest argues that if we take concepts to be research tools, we can understand them as applying provisionally 
or partially, and shift our attention from what concepts mean generally to what scientists take them to mean in particular cases.

In addition to the Focused Discussion section described above, which comprises a mix of invited and refereed short articles, this issue contains two full-length refereed articles. Both speak to the topic of scientific instruments, and I want to take special notice of them here. In the first article, MATTHEW SHINDELL examines the roles played by instruments such as the orbiting infrared spectrometer in the development of the field of planetary geology. Shindell finds that "a great deal of disciplinary work was necessary before field geologists could properly lay claim to planetary surfaces other than Earth's and before the instruments onboard the rovers could be considered geological tools" (p. 193). Instruments alone do not enable the study of phenomena. They need to be embedded within practices and user communities, who themselves understand these instruments within an existing (though adaptive) disciplinary frame. Moreover, "the instrument is not a simple extension of the senses of the scientist, but one part of a social project of perception" (p. 194). In the second article, DOROTHY SUTHERLAND OLSEN conceptualizes tools as "bearers of encapsulated knowledge and as being in a state where they can be both used and developed at the same time" (p. 250). This enables her to make sense of three different ways in which experimenters can gain knowledge: using tools according to established convention, using tools "wrongly," and the purposeful redesign of tools.

And what about that electron microscope in the hall? It is presently joined by dozens of other curated objects displayed and described with care and passion by an enterprising group of graduate students from IHPST. ERICH WEIDENHAMMER and MICHAEL DA SILVA describe the University of Toronto Scientific Instrument Collection project in our Opinion section. Aided by modest material (and wholehearted moral) institutional support, the UTSIC is the latest in a series of efforts to build a meaningful instrument collection at the University of Toronto.

\section{ACKNOWLEDGEMENTS}

My thanks to all of the contributors, who have made this Focused Discussion lively and enjoyable, and to the Institute for the History and Philosophy of Science and Technology, without whom this Journal could not exist. 


\author{
ISAAC RECORD \\ IHPST, University of Toronto \\ 91 Charles Street West \\ Toronto, Ontario M5S $1 \mathrm{~K} 7$ \\ isaac.record@utoronto.ca
}

\title{
REFERENCES
}

Baird, Davis. 2004. Thing Knowledge. Berkeley, CA: University of California Press.

Carroll-Burke, Patrick. 2001. Tools, Instruments, and Engines: Getting a Handle on the Specificity of Engine Science. Social Studies of Science 31(4): 593-625.

Galison, Peter. 1997. Image and Logic: a Material Culture of Microphysics. Chicago: Chicago University Press.

Hacking, lan. 1983. Representing and Intervening: Introductory Topics in the Philosophy of Natural Science. Cambridge: Cambridge University Press.

Humphreys, Paul. 2004. Extending Ourselves: Computational Science, Empiricism, and Scientific Method. Oxford: Oxford University Press.

Shapin, Steven and Simon Schaffer. 1985. Leviathan and the Air-Pump: Hobbes, Boyle, and the Experimental Life. Princeton, NJ: Princeton University Press.

Van Fraassen, Bas. 1980. The Scientific Image. Oxford: Oxford University Press. Warner, Deborah Jean. 1990. What is a Scientific Instrument, When did it Become One, and Why? British Journal for the History of Science 23(1): 83-93. 